\title{
Assignment of Fatty Acid- $\beta$-Oxidizing Syntrophic Bacteria to Syntrophomonadaceae fam. nov. on the Basis of 16S rRNA Sequence Analyses
}

\author{
HONGXUE ZHAO,${ }^{1}$ DECHENG YANG ${ }^{1}$ CARL R. WOESE, ${ }^{1}$ AND MARVIN P. BRYANT ${ }^{1,2 *}$ \\ Departments of Microbiology ${ }^{1}$ and Animal Sciences, ${ }^{2}$ University of Illinois, \\ Urbana, Illinois 61801
}

\begin{abstract}
After enrichment from Chinese rural anaerobic digestor sludge, anaerobic, sporing and nonsporing, saturated fatty acid- $\beta$-oxidizing syntrophic bacteria were isolated as cocultures with $\mathbf{H}_{2}$ - and formate-utilizing Methanospirillum hungatei or Desulfovibrio sp. strain G-11. The syntrophs degraded $\mathrm{C}_{4}$ to $\mathrm{C}_{8}$ saturated fatty acids, including isobutyrate and 2-methylbutyrate. They were adapted to grow on crotonate and were isolated as pure cultures. The crotonate-grown pure cultures alone did not grow on butyrate in either the presence or the absence of some common electron acceptors. However, when they were reconstituted with $M$. hungatei, growth on butyrate again occurred. In contrast, crotonate-grown Clostridium kluyveri and Clostridium sticklandii, as well as Clostridium sporogenes, failed to grow on butyrate when these organisms were cocultured with $M$. hungatei. The crotonate-grown pure subcultures of the syntrophs described above were subjected to 16S rRNA sequence analysis. Several previously documented fatty acid- $\beta$-oxidizing syntrophs grown in pure cultures with crotonate were also subjected to comparative sequence analyses. The sequence analyses revealed that the new sporing and nonsporing isolates and other syntrophs that we sequenced, which had either gram-negative or gram-positive cell wall ultrastructure, all belonged to the phylogenetically gram-positive phylum. They were not closely related to any of the previously known subdivisions in the gram-positive phylum with which they were compared, but were closely related to each other, forming a new subdivision in the phylum. We recommend that this group be designated Syntrophomonadaceae fam. nov.; a description is given.
\end{abstract}

In the last few years it has been shown that anaerobic saturated fatty acid- $\beta$-oxidizing syntrophs (SFAS) can be adapted to grow with the unsaturated acid crotonate as the energy source and then purified by single-colony isolation ( 5 , 44). Using two pure cultures, we initiated a phylogenetic study of SFAS by 16 S rRNA sequences analysis $(18,44)$. Syntrophomonas wolfei subsp. wolfei is nonsporing and has a gram-negative cell wall ultrastructure, and Syntrophospora bryantii is a gram-positive sporeformer. Both of these organisms belong to the phylogenetically gram-positive phylum, and they are much more closely related to each other than they are to any other member of the gram-positive phylum with which they have been compared (44).

In the present study, cocultures of three new strains of SFAS were isolated and characterized. These syntrophic strains plus another syntroph, NSF-2 (31), were isolated in pure cultures by using crotonate as the energy source. All of the pure subcultures of the SFAS available so far (four obtained in the present study and three obtained from previous studies $[5,18,44])$ were subjected to comparative $16 \mathrm{~S}$ rRNA sequence analyses. The results revealed not only that all of the mesophilic SFAS were unique in their syntrophic metabolism but also that these organisms formed a new Syntrophomonas subdivision in the phylogenetically gram-positive phylum. In addition, Clostridium kluyveri, a well-known crotonate catabolizer, and two other species of the genus Clostridium could not be adapted to behave as syntrophic fatty acid degraders.

\footnotetext{
${ }^{*}$ Corresponding author.
}

\section{MATERIALS AND METHODS}

Source for enrichment. A sample of rural domestic digestor sludge was collected in Chengdu, People's Republic of China, by Minrong Zhang of the Chengdu Biogas Research Institute of the Chinese Ministry of Agriculture. The sample was transferred into a glass container, sealed, and stored at room temperature for 1 week and at $4^{\circ} \mathrm{C}$ for 4 months before processing.

Strains. Desulfovibrio sp. strain G-11 (21) and Methanospirillum hungatei JF1 were obtained from the culture collection of M. P. Bryant. Syntrophic strain NSF-2 (31) in triculture with Methanosarcina barkeri and Methanospirillum hungatei (37) was a gift from J. M. Tiedje, Michigan State University, East Lansing. Methanospirillum hungatei SK was a gift from F. Widdel, Philipps-Universität, Marburg, Germany. C. kluyveri ATCC 8527 and Clostridium sporogenes ATCC 3584 were obtained from the American Type Culture Collection, Rockville, Md. Clostridium sticklandii VPI 12497B was a gift from W. E. C. Moore, Virginia Polytechnic Institute and State University, Blacksburg. Escherichia coli K-12 was a gift from Shyr-jiann Li, University of Illinois, Urbana.

Cultivation conditions. The anaerobic techniques used for the preparation and use of the media included both the Hungate technique (14) as modified by Bryant (8) and the technique of Balch and Wolfe (2). The basal medium was as described previously (21). Unless otherwise indicated, liquid media supplemented with different substrates and other special additives were prepared by addition of sterile anaerobic stock solutions to individual serum tubes (18 by 150 $\mathrm{mm}$ ) containing the basal medium by means of syringes prior to inoculation. The reducing agent $\mathrm{Na}_{2} \mathrm{~S} \cdot 9 \mathrm{H}_{2} \mathrm{O}$ was added in the same manner. Media supplemented with insoluble 
fatty acids were prepared as described previously (18). Sterile anaerobic stock solutions of 3-hydroxybutyrate, 2-bromoethanesulfonic acid (3), and amino acids were prepared by filter sterilization under $\mathrm{O}_{2}$-free $\mathrm{N}_{2}$ and stored at $-20^{\circ} \mathrm{C}$. Solid media for roll tubes and slants were prepared as described previously (21). Liquid cultures of Desulfovibrio sp. strain G-11 and Methanospirillum hungatei were grown under a $\mathrm{H}_{2}-\mathrm{CO}_{2}$ gas phase, as described previously (21). Syntrophic strain NSF-2 in triculture was maintained in liquid butyrate medium and transferred monthly. All cultures containing SFAS (including the enrichment cultures) were incubated in the dark at $36^{\circ} \mathrm{C}$. Cell masses needed for isolation of RNA and DNA were cultivated in 2-liter serum bottles (24). The culture tubes which did not show growth after 6 weeks but needed to be incubated longer were further incubated in anaerobic incubation vessels (17) with a gas phase containing $\mathrm{N}_{2}$ and $\mathrm{CO}_{2}(1: 4,200 \mathrm{kPa})$.

Enrichment and isolation. Butyrate-degrading enrichment cultures were initiated in serum tubes containing $1 \mathrm{~g}$ of Chinese sludge sample in $10 \mathrm{ml}$ of the basal medium supplemented with $20 \mathrm{mM}$ butyrate. In the control tubes butyrate was absent. The primary enrichment cultures which showed good methane production were subjected to successive $50 \%$ (vol/vol) transfers into fresh butyrate medium until the enrichment cultures became stable with respect to the extent and the rate of butyrate degradation and methane production and with respect to the microscopically observed microbial population.

Isolation of SFAS in coculture with an $\mathrm{H}_{2}$-formate utilizer by the roll tube method was performed as described previously (21). Active liquid cultures of Methanospirillum hungatei JF-1 or Desulfovibrio sp. strain G-11 were used as lawns of the $\mathrm{H}_{2}$ and formate utilizer. Isolation of pure subcultures of SFAS was performed by the roll tube method with $20 \mathrm{mM}$ crotonate medium.

Maintenance and purity control. The newly isolated cocultures were maintained in stabbed slants of basal medium supplemented with $20 \mathrm{mM}$ butyrate. In the coculture with Desulfovibrio sp., $20 \mathrm{mM} \mathrm{Na}_{2} \mathrm{SO}_{4}$ was also included. Transfers were made every 2 months. The purity of the isolates was checked by microscopic examination of wet mounts and by inoculating samples into basal medium supplemented with $0.1 \%$ (wt/vol) glucose, $0.1 \%$ (wt/vol) cellobiose, and $0.1 \%$ (wt/vol) starch (GCS medium) and incubating the preparations under aerobic and anaerobic conditions, which allowed no growth of the SFAS.

Electron microscopy. Cells of young cultures were centrifuged, and the pellets were fixed with $2.6 \%$ glutaraldehyde in $0.1 \mathrm{M}$ cacodylate buffer at $4^{\circ} \mathrm{C}$. Postfixation was done with $1 \%$ osmium tetroxide and $1.5 \%$ potassium ferricyanide at room temperature. The samples were stained en bloc with $2 \%$ uranyl acetate in $10 \%$ ethanol and washed with water. Dehydration was achieved by using acidified dimethoxypropane and then acetone. The samples were infiltrated with mixtures containing acetone and epoxy (1:1 and then 1:3) and finally with pure epoxy. The cells were spun down in epoxy-filled Beem capsules and polymerized at $90^{\circ} \mathrm{C}$. Ultrathin sections were photographed at $80 \mathrm{kV}$ with a JOEL 100 CX transmission electron microscope (23). To observe flagella, samples for negative staining were prepared by floating a pair of Formvar ( $0.25 \%$ in ethylene dichloride)-coated copper grids on a drop of a bacterial suspension for $15 \mathrm{~min}$. The grids were negatively stained, one with $2 \%$ ammonium molybdate and the other with $2 \%$ phosphotungstic acid (4).

Light microscopy. Both phase-contrast microscopy and epifluorescence microscopy were used for routine purposes.
Phase-contrast photomicrographs were taken by using wet mounts on agar-coated slides (27).

Determination of growth and fermentation products. Growth was routinely followed by measuring $A_{600}$ and by measuring substrate consumption and product generation by high-performance liquid chromatography (HPLC) and gas chromatography, as described below. Cell numbers were estimated by using a Petroff-Hausser counting chamber. Total gas volume was determined by displacement of the plunger of a glass piston syringe. Gas was analyzed for $\mathrm{CH}_{4}$ and $\mathrm{H}_{2}$ gas chromatography (21). Organic acids, mainly volatile fatty acids, were determined as free acids by HPLC (10) and sometimes were determined as butyl esters by gas chromatography (29). Oxygen sensitivity was tested in stabbed slants (9).

Test for substrate utilization. Utilization of energy sources by the SFAS was tested by inoculating $0.4-\mathrm{ml}$ portions of an active butyrate-degrading coculture into serum tubes containing $10 \mathrm{ml}$ of basal medium without and with the compound of interest at the test concentration. After 30 days of incubation, the culture tubes which did not show growth were incubated in anaerobic incubation vessels as described above, and the growth was then checked weekly for up to 90 days.

Test for electron acceptors. Butyrate $(20 \mathrm{mM})$ was present as the electron donor. For the nonsporing isolate cocultured with a methanogen, the test was performed in the presence and absence of 2-bromoethanesulfonic acid. For the sporing isolates, sporulated, pasteurized $\left(80^{\circ} \mathrm{C}, 10 \mathrm{~min}\right)$ cocultures were used as the inocula.

Determination of DNA base composition. DNA was isolated from crotonate-grown pure subcultures of the SFAS. The $\mathrm{G}+\mathrm{C}$ content of the DNA was determined by the thermal denaturation method by using a Gilford Response spectrophotometer equipped with a thermoprogram, as described previously (44).

$16 S$ rRNA sequencing. The crotonate-grown subcultures of the SFAS were used for isolation of RNA, as described previously (44). An unfractionated cellular RNA preparation was used directly for sequencing. The 16S rRNA sequence was determined by a modification of the standard Sanger dideoxynucleotide chain termination method (30), using avian myeloblastosis virus reverse transcriptase and synthetic oligodeoxynucleotide primers complementary to conserved regions of $16 \mathrm{~S}$ rRNA (16). Eight or more primers were used (43). The entire $16 \mathrm{~S}$ rRNA molecule except for about 60 bases at the $3^{\prime}$ end was sequenced.

Sequence analysis. The sequences of the syntrophic bacteria were aligned by using a standard method (42) with the sequences representing different phylogenetic clusters of gram-positive eubacteria. Sequences of some gram-negative bacteria were used as outgroups. The sequence similarities and the evolutionary distances were calculated by using the method of Jukes and Cantor (15). Only those positions in the alignment represented by known bases in all sequences represented were used in the calculation. A phylogenetic tree was constructed from the evolutionary distances by using the algorithm of De Soete (12).

Nucleotide sequence accession numbers. The GenBank accession numbers for the partial 16S rRNA sequences of the various bacteria used (see Table 3 and Fig. 4) were as follows: Syntrophomonas wolfei, M26492; Syntrophospora bryantii, M26491; Clostridium butyricum, M59085; Clostridium pasteurianum, M23930; C. sporogenes, M59115; C. kluyveri, M59092; Clostridium aminovalericum, M23929; Bacillus subtilis, J01551; Clostridium barkeri, M23927; 
Sporomusa paucivorans, M59117; Clostridium quercicolum, M59110; Megasphaera elsdenii, M26493; Heliobacterium clorum, M11212; Agrobacterium globiformis, M23411; and Desulfovibrio desulfuricans, M34113. Syntroph strains FSM2 and FSS7 were lost before they could be deposited in a culture collection, and therefore, they could not be named and their 16S rRNA partial sequences could not be deposited in the GenBank data base; however, their sequences are available from C.R.W.

\section{RESULTS}

Enrichment. Within 2 weeks of inoculation, gas production was visible, and $\mathrm{CH}_{4}$ was detected in the primary enrichment cultures. After 4 months of successive transfers, the enrichment cultures were stabilized, with butyrate converted into $\mathrm{CH}_{4}$ and acetate at a stable rate, and the following microbial morphotypes were present: (i) a large number of autoflourescent, motile, long rods morphologically identical to Methanospirillum hungatei (morphotype I); (ii) a large number of nonfluorescent, nonmotile, large, filamentous cells morphologically identical to Methanothrix sp. (morphotype II); and (iii) smaller numbers of nonfluorescent, nonmotile, slightly curved or straight medium-size rods with variable Gram reactions (morphotype III). Endospores were observed in some morphotype III cells.

Coculture isolation. For the roll tubes with lawns of strain G-11, dark round colonies 1 to $2 \mathrm{~mm}$ in diameter developed only in the roll tubes containing butyrate after 3 to 4 weeks of incubation. After 6 weeks of incubation, well-separated colonies were picked into slants, and growth occurred. In addition to actively motile cells of strain G-11, each of the slants contained a second morphotype, consisting of nonmotile, slightly curved or straight rods exhibiting variable Gram staining. In some of the isolates, the second morphotype formed endospores. These slant cultures were transferred to the liquid medium and then subjected to a second set of roll tube coculture isolations. The resulting morphotypes were the same as those in the first set of slants. For the roll tubes with lawns of Methanospirillum hungatei JF1, the final resulting morphotypes of the butyrate degraders were the same as those isolated with strain G-11, except that the colonies of the cocultures were yellowish and it took more than 4 weeks for the colonies to develop.

All of the new isolates were subjected to purity examination with GCS medium. Three of the pure cocultures were chosen for further study.

Morphology. Strain FSM2, cocultured with Methanospirillum hungatei JF-1, was a gram-variable, sporing, curved, rod-shaped organism ( 0.4 by 1 to $2 \mu \mathrm{m})$ that occurred singly and in pairs. It had oval to oblong terminal to subterminal spores that swelled the sporangia. Motility was not detected. Cells tended to clump but were easily dispersed by shaking. Strain FSS7, cocultured with Desulfovibrio sp. strain G-11, was an almost straight sporeformer that otherwise was very similar to strain FSM2. Strain FM4, cocultured with Methanospirillum hungatei JF-1, was a nonsporing, straight, rod-shaped organism that otherwise was very similar to the sporing strains. Electron micrographs of thin sections of the three strains revealed similar cell membranes, a relatively thin layer of peptidoglycan, a surface slime layer, and no outer membrane (Fig. 1).

Substrate utilization. The three cocultures all grew on fourto eight-carbon saturated fatty acids but not on fatty acids containing three or more than eight carbon atoms (Table 1). Strain FSS7 showed slight growth on Casamino Acids (Table
1), but it failed to grown on individual amino acids and pairs of amino acids known to support the growth of some Clostridium species that carry out the Stickland reaction (26).

Isolation of SFAS in pure cultures. The three cocultures, FSS7, FSM2, and FM4, and NSF-2 in triculture were all adapted to grow on the basal medium supplemented with 20 $\mathrm{mM}$ crotonate or $20 \mathrm{mM} 3$-hydroxybutyrate. After successive transfers in the crotonate medium, the $\mathrm{H}_{2}$ - and formateutilizing partner became a much smaller proportion of the total cells in each of the cultures. After prolonged incubation, endospores appeared in the crotonate-grown FSS7 and FSM2 cultures, but the ratios of sporulated cells to unsporulated cells were much lower than when the organisms were grown in the butyrate medium; in contrast to the cocultures on butyrate, the sporulated cultures did not survive pasteurization.

The crotonate-grown coculture of strains FSM2 and JF-1 was successively transferred four times in crotonate medium containing $200 \mu \mathrm{M}$ 2-bromoethanesulfonic acid. In the culture after the last transfer, only a few cells of Methanospirillum hungatei JF1 were observed, and they were ghostlike. This culture was then subjected to roll tube isolation on crotonate medium. Well-separated colonies were transferred into liquid crotonate medium; they all grew and had the cell morphology of FSM2. After examination for contamination with GCS medium, one of the pure isolates, designated strain FSM2C, was used for further studies. Pure subcultures FM4C and NSF-2C were also used for further studies; these pure subcultures were isolated in the same way. Attempts to obtain highly purified FSS7 by molybdate inhibition of the Desulfovibrio sp. cells (32) in the coculture of FSS7 and G-11 were unsuccessful. Pure subculture FSS7C was isolated on crotonate roll tubes without pretreatment by 2 -bromoethanesulfonic acid. Figure 2 shows the time course of crotonate degradation into the major products acetate and butyrate by strain FSS7C. Crotonate fermentation by the three pure subcultures was similar to crotonate fermentation by a pure subculture of Syntrophomonas wolfei subsp. wolfei (5), except that the three pure subcultures produced higher ratios of butyrate to acetate (Table 2).

Test for butyrate utilization by the pure subcultures. Within 90 days of inoculation, strains FSS7C, FSM2C, and FM4C did not grow in $20 \mathrm{mM}$ butyrate medium in the presence or absence of sulfate $(20 \mathrm{mM})$, nitrate $(10 \mathrm{mM})$, and fumarate (20 $\mathrm{mM})$, respectively.

Reconstitution test. Strains FSS7C, FSM2C, and FM4C were each reconstituted with Methanospirillum hungatei SK in butyrate medium along with controls lacking the methanogen or butyrate, as described previously (44). Growth occurred only in the tubes containing both butyrate and Methanospirillum hungatei. Figure 3 shows the time course of butyrate degradation by the stabilized coculture of strains FSS7C and SK. The results were very similar with the reconstituted cocultures of strains FSM2C and SK and strains FM4C and SK. When the stabilized cocultures of strains FSS7C and SK and strains FSM2C and SK were incubated for 7 weeks, spores appeared, and the ratio of sporulated cells to unsporulated cells was again much higher than the ratio in the previous cultures grown on crotonate. These results demonstrated that the three crotonate-grown pure subcultures behaved as syntrophic butyrate degraders, as did the original syntrophs.

Some growth parameters. Strains FSM2C, FSS7C, and FM4C were anaerobic as they failed to grow in the crotonate medium when a little $\mathrm{O}_{2}$ was added to the culture tubes by 


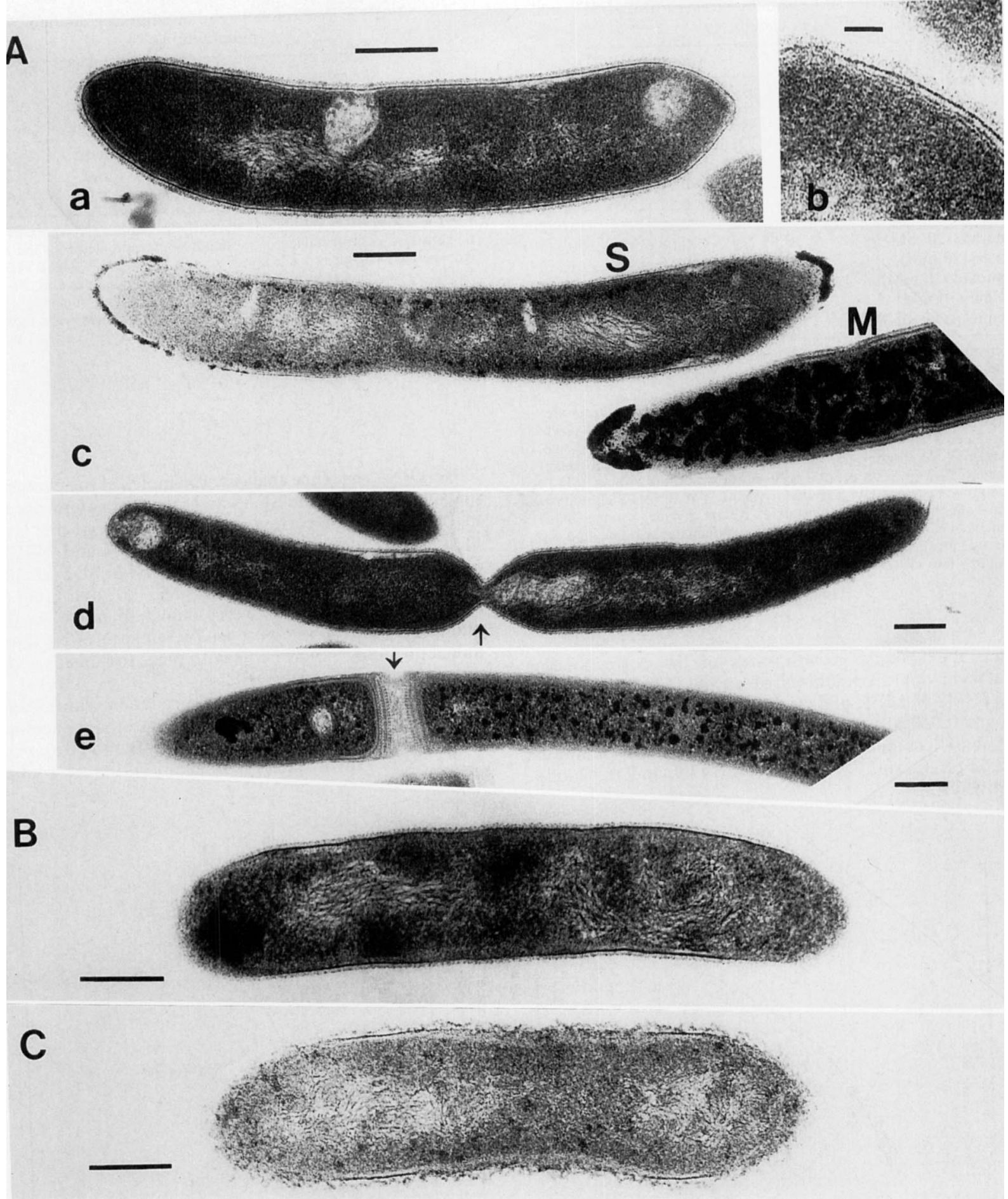

FIG. 1. Electron micrographs of ultrathin sections of strains FSM2, FSS7, and FM4. (Aa) Cell of strain FSM2. (Ab) Section of the cell wall and membranes of FSM2. Bar $=50 \mathrm{~nm}$. (Ac) Strain FSM2 and its syntrophic partner Methanospirillum hungatei. Note the difference in the cell walls and membranes of FSM2 (S) and Methanospirillum hungatei (M). (Ad and Ae) Comparison of the transverse septa and walls of dividing cells of FSM2 (Ad) and Methanospirillum hungatei (Ae). The arrows indicate the cell junctions. (B) Cell of strain FSS7. (C) Cell of strain FM4. The bars in all panels except panel $\mathrm{Ab}$ are $250 \mathrm{~nm}$ long.

means of a syringe (resazurin in the medium turned pink). They all grew at crotonate concentrations ranging from 5 to $45 \mathrm{mM}$, but in contrast to Syntrophomonas wolfei (5), they did not grow in medium containing $60,70,80$, or $90 \mathrm{mM}$ crotonate. They grew at 25,36 , and $46^{\circ} \mathrm{C}$, but not at 50 or $20^{\circ} \mathrm{C}$. At $36^{\circ} \mathrm{C}$ in $20 \mathrm{mM}$ crotonate medium the specific growth rates for strains FSS7C, FSM2C, and FM4C were $0.022,0.025$, and $0.020 \mathrm{~h}^{-1}$, respectively. In $20 \mathrm{mM}$ butyrate 
TABLE 1. Substrate utilization by strains FSM2, FSS7, and FM4 in cocultures ${ }^{a}$

\begin{tabular}{llll}
\hline \multirow{2}{*}{$\begin{array}{c}\text { Substrate added to } \\
\text { basal medium }\end{array}$} & \multicolumn{3}{c}{ Growth $\left(A_{600}\right)$} \\
\cline { 2 - 4 } & Strain FSM2 & Strain FSS7 & Strain FM4 \\
\hline None (basal medium control) & $0.02(6)^{b}$ & $0.01(5)$ & $0.02(6)$ \\
Propionate $(20 \mathrm{mM})$ & $0.02(7)$ & $0.02(8)$ & $0.02(7)$ \\
Butyrate $(20 \mathrm{mM})$ & $0.23(14)$ & $0.31(16)$ & $0.20(16)$ \\
Isobutyrate $(20 \mathrm{mM})$ & $0.12(17)$ & $0.17(17)$ & $0.09(18)$ \\
2-Methylbutyrate $(20 \mathrm{mM})$ & $0.10(17)$ & $0.11(17)$ & $0.08(19)$ \\
Valerate $(20 \mathrm{mM})$ & $0.09(19)$ & $0.12(19)$ & $0.10(20)$ \\
Isovalerate $(20 \mathrm{mM})$ & $0.09(19)$ & $0.08(19)$ & $0.07(19)$ \\
Caproate $(20 \mathrm{mM})$ & $0.14(16)$ & $0.15(18)$ & $0.11(17)$ \\
Heptanoate $(10 \mathrm{mM})$ & $0.06(20)$ & $0.07(20)$ & $0.06(20)$ \\
Caprylate $(10 \mathrm{mM})$ & $0.09(18)$ & $0.09(19)$ & $0.08(18)$ \\
Casamino Acids $(0.05 \%)$ & $0.02(6)$ & $0.05(9)$ & $0.02(6)$ \\
\hline
\end{tabular}

${ }^{a}$ Cultivation was performed in triplicate in serum tubes (18 by $150 \mathrm{~mm}$ ) containing $10 \mathrm{ml}$ of medium. Each inoculum was $6 \%$ of a butyrate-grown young coculture.

${ }^{b}$ Means of two sets of triplicate tubes. The numbers in parentheses are the incubation times (in days) required to reach the maximum $A_{600}$. No products were produced from $4 \mathrm{mM}$ decanoate, $3 \mathrm{mM}$ tridecanoate, $3 \mathrm{mM}$ palmitate, or $2 \mathrm{mM}$ stearate. No growth occurred with $10 \mathrm{mM}$ glucose, $10 \mathrm{mM}$ fructose, 10 $\mathrm{mM}$ mannitol, $0.05 \%$ Casitone, or $0.05 \%$ yeast extract. For strains FSM2 and FM4, no growth occurred with $10 \mathrm{mM}$ pyruvate. For strain FSS7, no growth occurred with the individual amino acids lysine, glycine, proline, alanine, aspartate, valine, leucine, isoleucine, cystine, glutamate, histidine, and serine (each at a concentration of $50 \mathrm{mM}$ ) and with pairs of amino acids known to support growth of Clostridium species that carry out the Stickland reaction.

medium, the specific growth rates of the corresponding original syntrophic cocultures of strains FSS7 and G-11, strains FSM2 and JF1, and strains FM4 and JF1 were 0.014, 0.013 , and $0.010 \mathrm{~h}^{-1}$, respectively.

DNA base compositions. The $\mathrm{G}+\mathrm{C}$ contents of strains FSM2C, FSS7C, and FM4C were 44.6, 47.9, and $41.1 \mathrm{~mol} \%$, respectively.

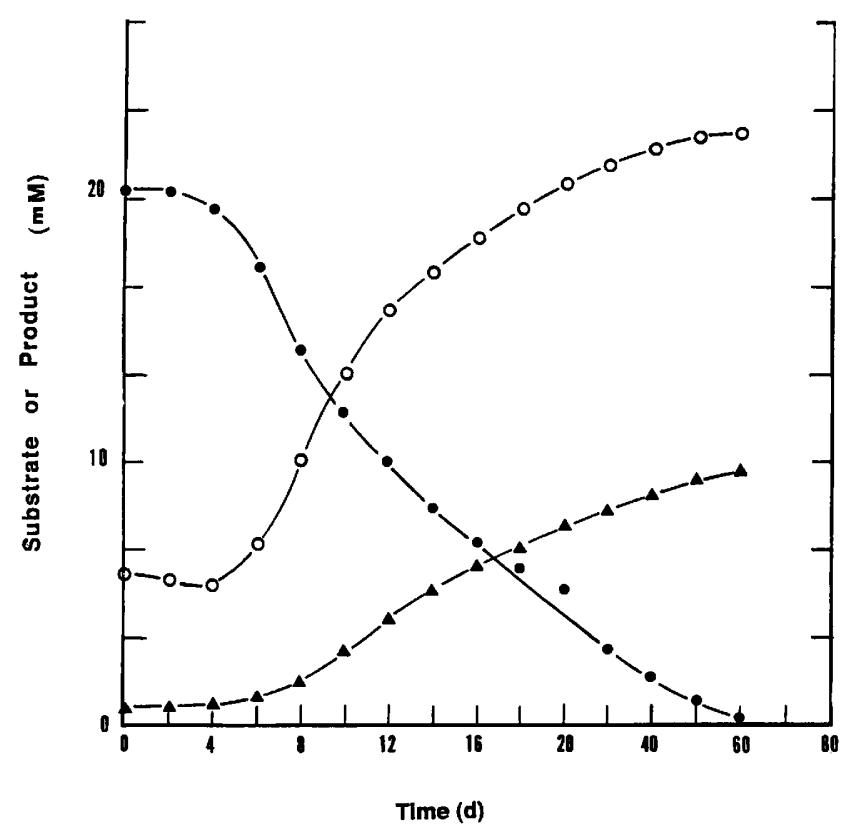

FIG. 2. Time course of crotonate conversion to the major end products acetate and butyrate by a pure subculture of strain FSS7C. $\boldsymbol{Q}$, crotonate; $O$, acetate; $\boldsymbol{\Delta}$, butyrate. d, days.
TABLE 2. Crotonate and butyrate degradation by pure cultures

\begin{tabular}{|c|c|c|c|c|c|c|}
\hline \multirow[t]{2}{*}{ Inoculum } & \multirow{2}{*}{$\begin{array}{l}\text { Concn of } \\
\text { crotonate } \\
(\mathrm{mM})\end{array}$} & \multirow{2}{*}{$\begin{array}{l}\text { Growth } \\
\left(A_{600}\right)\end{array}$} & \multicolumn{2}{|c|}{$\begin{array}{l}\text { Concn of major } \\
\text { products }(\mathrm{mM})\end{array}$} & \multicolumn{2}{|c|}{$\begin{array}{l}\text { \% Recovery } \\
\text { of: }\end{array}$} \\
\hline & & & Acetate & Butyrate & C & $\mathrm{H}$ \\
\hline SM2C & 19.7 & 0.42 & & 6.4 & 80.7 & 79.0 \\
\hline FSS7C & 20. & 0.48 & 19.1 & 6.9 & 81.4 & 80.0 \\
\hline FM4C & 20.5 & 0.36 & 19.4 & 6.2 & 77.6 & 75.7 \\
\hline
\end{tabular}

${ }^{a}$ The values are means of two sets of triplicate tubes and were corrected for no-substrate control values. The amounts of caproate, $\mathrm{H}_{2}$, and substrate incorporated into ceils were not determined. Strains FSM2C, FSS7C, and FM4C were also tested with $20 \mathrm{mM}$ butyrate medium in the absence and presence of sulfate $(20 \mathrm{mM})$, nitrate $(10 \mathrm{mM})$, and fumarate $(20 \mathrm{mM})$, respectively. After 30 days of incubation, these cultures were further incubated in anaerobic incubation vessels as described in the text; no growth occurred after 13 weeks of incubation.

16S rRNA sequence analysis. Examples of the evolutionary distances between the SFAS and other eubacteria are shown in Table 3, and the corresponding phylogenetic tree is shown in Fig. 4. The sequences of strains FM4 and NSF-2 and Syntrophomonas wolfei subsp. saponavida SD2 (18) derived from four primers (positions 519, 690, 907, and 1100) (Escherichia coli numbering) were almost identical to the sequence of Syntrophomonas wolfei subsp. wolfei; thus, further sequencing experiments with these organisms and other primers were not performed.

Test for syntrophic butyrate degradation with Clostridium spp. C. kluyveri ATCC 8527 grown on ethanol-acetate medium (36) and $C$. sticklandii VPI 12497B and $C$. sporogenes ATCC 3584 grown on PYG medium (11) were transferred

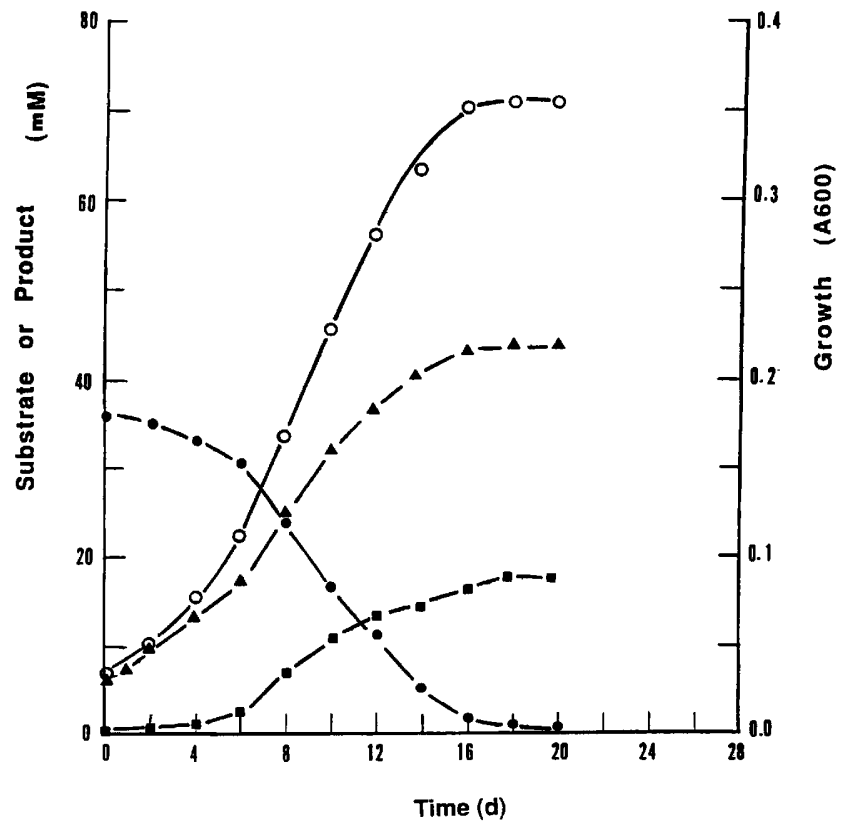

FIG. 3. Time course of butyrate degradation by a stabilized reconstituted coculture of strain FSS7C and Methanospirillum hungatei SK. - butyrate concentration; $\bigcirc$, acetate concentration; $\mathbf{\square}$, methane concentration; $\boldsymbol{A}$, growth. $\mathrm{d}$, days. 
TABLE 3. Evolutionary distances

\begin{tabular}{|c|c|c|c|c|c|c|c|c|c|c|c|c|c|c|c|c|}
\hline \multirow[b]{2}{*}{ Organism } & \multicolumn{16}{|c|}{ Evolutionary distances } \\
\hline & 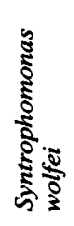 & 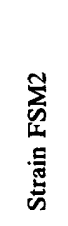 & 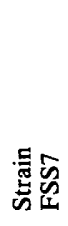 & 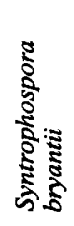 & 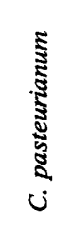 & 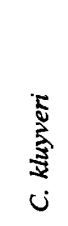 & 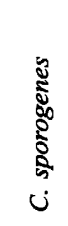 & 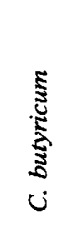 & 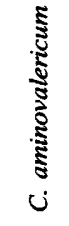 & $\begin{array}{l}5 \\
\text { है } \\
0 \\
0\end{array}$ & 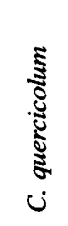 & 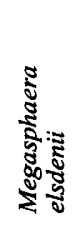 & 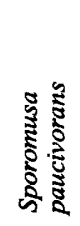 & 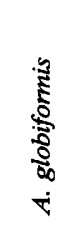 & 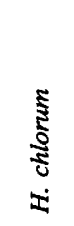 & 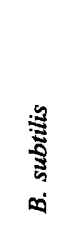 \\
\hline Strain FSM2 & 6.7 & & & & & & & & & & & & & & & \\
\hline Strain FSS7 & 5.3 & 3.6 & & & & & & & & & & & & & & \\
\hline Syntrophospora bryantii & 5.4 & 6.0 & 4.7 & & & & & & & & & & & & & \\
\hline C. pasteurianum & 19.2 & 18.2 & 18.3 & 19.1 & & & & & & & & & & & & \\
\hline C. kluyveri & 19.9 & 19.6 & 19.0 & 19.2 & 10.1 & & & & & & & & & & & \\
\hline C. sporogenes & 16.8 & 16.8 & 16.4 & 16.4 & 8.5 & 11.7 & & & & & & & & & & \\
\hline C. butyricum & 19.2 & 18.7 & 17.4 & 17.8 & 7.1 & 11.2 & 8.4 & & & & & & & & & \\
\hline C. aminovalericum & 18.5 & 18.6 & 17.8 & 18.1 & 18.5 & 18.7 & 18.6 & 18.7 & & & & & & & & \\
\hline C. barkeri & 20.4 & 20.2 & 19.8 & 20.4 & 19.2 & 17.4 & 18.9 & 20.4 & 20.6 & & & & & & & \\
\hline C. quercicolum & 21.1 & 19.5 & 19.4 & 21.3 & 21.0 & 21.0 & 22.2 & 23.4 & 22.8 & 21.8 & & & & & & \\
\hline Megasphaera elsdenii & 22.1 & 21.4 & 20.8 & 22.3 & 22.5 & 24.6 & 23.4 & 23.9 & 22.1 & 24.4 & 12.7 & & & & & \\
\hline Sporomusa paucivorans & 22.1 & 19.6 & 20.3 & 21.7 & 20.4 & 22.1 & 21.7 & 22.2 & 22.9 & 23.2 & 10.0 & 16.0 & & & & \\
\hline A. globiformis & 23.9 & 21.4 & 22.3 & 23.4 & 23.3 & 25.0 & 24.3 & 23.6 & 21.7 & 22.1 & 24.2 & 25.4 & 24.0 & & & \\
\hline H. chlorum & 18.2 & 17.6 & 17.7 & 18.5 & 17.7 & 19.8 & 18.7 & 19.8 & 22.1 & 21.3 & 16.7 & 21.0 & 18.2 & 21.5 & & \\
\hline B. subtilis & 17.9 & 18.6 & 17.8 & 19.0 & 17.9 & 18.7 & 18.2 & 19.0 & 19.9 & 17.0 & 19.1 & 22.5 & 19.2 & 20.6 & 19.0 & \\
\hline D. desulfuricans & 21.8 & 20.4 & 19.8 & 19.9 & 23.3 & 24.3 & 24.0 & 24.1 & 21.8 & 23.0 & 25.4 & 25.1 & 26.3 & 23.9 & 21.7 & 22.3 \\
\hline
\end{tabular}

into the crotonate medium. Only the first two grew. Young cultures of $C$. kluyveri and $C$. sticklandii grown on the crotonate medium and $C$. sporogenes grown on PYG medium were each combined with an active culture of Metha- nospirillum hungatei SK grown on $\mathrm{H}_{2}-\mathrm{CO}_{2}$ in $20 \mathrm{mM}$ butyrate medium by using the same method used in the reconstitution tests. No growth was observed after 90 days of incubation. The same experiments were repeated three

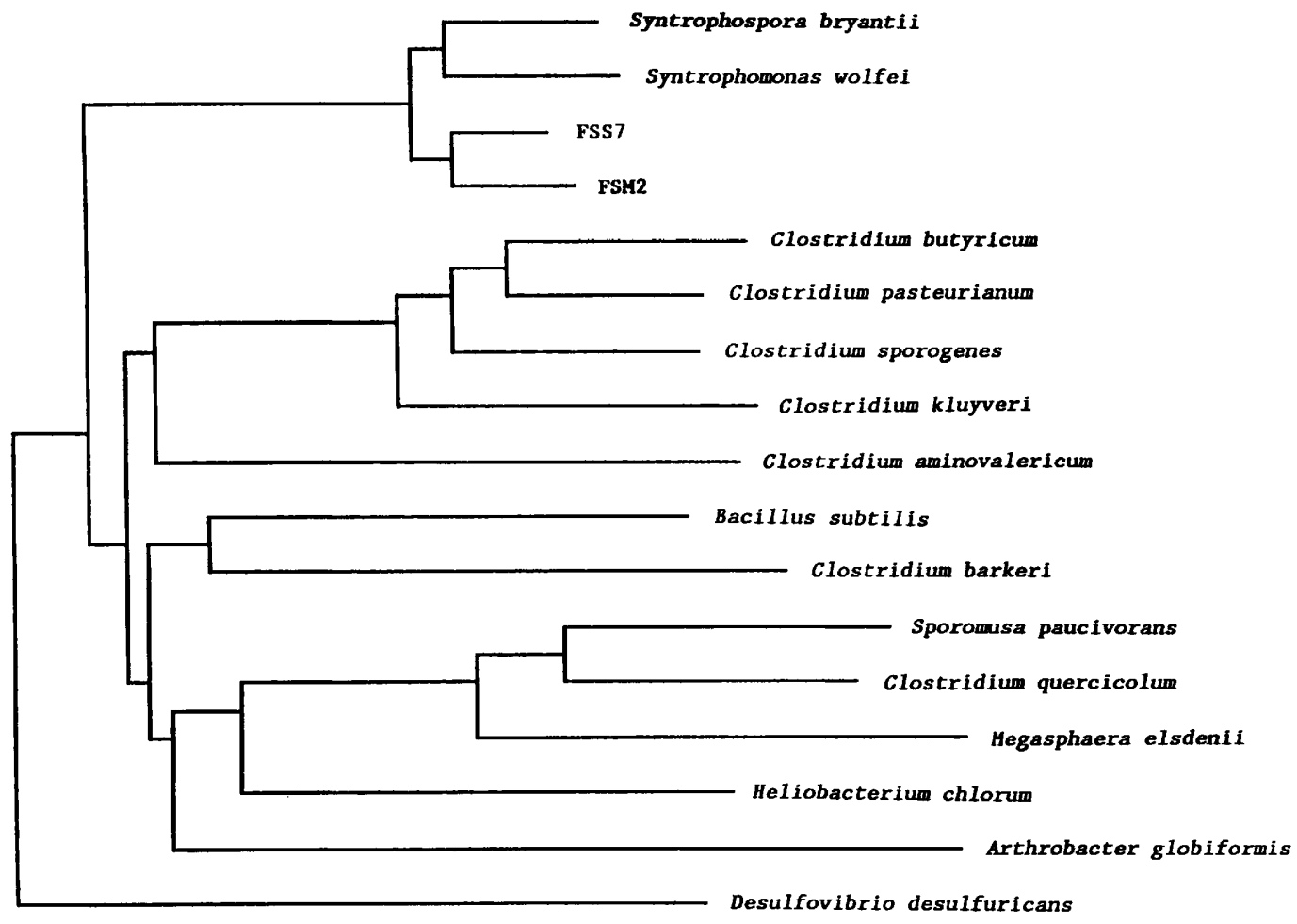

FIG. 4. Phylogenetic relationships of mesophilic syntrophic fatty acid- $\beta$-oxidizing bacteria. The phylogenetic tree was constructed by using the evolutionary distances shown in Table 3 and the algorithm of De Soete (12). Bar $=0.05$ evolutionary distance unit. 
TABLE 4. Comparison of the characteristics of the mesophilic SFAS

\begin{tabular}{|c|c|c|c|c|c|c|c|c|}
\hline \multirow[b]{2}{*}{ Taxon } & \multirow[b]{2}{*}{ Spores } & \multirow{2}{*}{$\begin{array}{l}\text { Cell outer } \\
\text { membrane }\end{array}$} & \multirow{2}{*}{$\begin{array}{c}\mathrm{G}+\mathrm{C} \\
\text { content } \\
(\text { mol } \%)\end{array}$} & \multicolumn{4}{|c|}{ Catabolism of: } & \multirow[b]{2}{*}{ Reference(s) } \\
\hline & & & & Isobutyrate & $\begin{array}{l}\text { 2-Methyl- } \\
\text { butyrate }\end{array}$ & $\begin{array}{l}\mathrm{C}_{9} \text { to } \mathrm{C}_{11} \\
\text { fatty acids }\end{array}$ & $\begin{array}{l}\mathrm{C}_{12} \text { to } \mathrm{C}_{18} \\
\text { fatty acids }\end{array}$ & \\
\hline FM4 & - & - & 41.1 & + & + & - & - & This study \\
\hline FMS2 & + & - & 47.9 & + & + & - & - & This study \\
\hline FSS7 & + & - & 44.6 & + & + & - & - & This study \\
\hline Syntrophospora bryantii & + & - & 37.6 & - & + & + & - & 34,44 \\
\hline $\begin{array}{l}\text { Syntrophomonas wolfei } \\
\text { subsp. wolfei }\end{array}$ & - & + & $\mathrm{ND}^{a}$ & - & - & - & - & 20,44 \\
\hline $\begin{array}{l}\text { Syntrophomonas wolfei } \\
\text { subsp. saponavida }\end{array}$ & - & ND & ND & - & - & + & + & 18 \\
\hline NSF-2 & - & ND & ND & ND & + & ND & ND & 31 \\
\hline
\end{tabular}

${ }^{a} \mathrm{ND}$, not determined.

times, and the same results were obtained. This indicated that these Clostridium spp. could not behave as SFAS.

\section{DISCUSSION}

The three new isolates and all other SFAS which we sequenced had two important phenotypic features in common, a unique syntrophic saturated fatty acid metabolism and the ability to metabolize crotonate in pure culture (Table 4). They differed in some aspects of their morphology, such as cell wall ultrastructure and spore formation. Also, they had more or less different substrate ranges and DNA base compositions (Table 4).

Of particular interest is the fact that all of the SFAS which we sequenced, including those having either gram-negative or gram-positive ultrastructure (44), belonged to the phylogenetically gram-positive phylum (41). However, they were not closely related to any of the members of the subdivisions in the gram-positive phylum with which they were compared (Fig. 4). On the other hand, they were all closely related to each other, forming a new subdivision in the gram-positive phylum.

We know of no well-documented bacterium located outside the new subdivision that can function as an SFAS. For example, neither the well-known crotonate catabolizer $C$. kluyveri (36), the other two Clostridium species included in this study, nor the fatty acid-degrading sulfate reducers (40) behave like SFAS. The crucial difference in metabolism between the syntrophic and nonsyntrophic bacteria appeared to reflect their phylogenetic differences.

The sequence analyses also indicated that not only within the new subdivision but also in comparisons between this subdivision and some other subdivisions of the gram-positive phylum, the Gram staining characteristics, the cell wall ultrastructure (presence or absence of a outer cell membrane), the ability to form spores, the presence or absence of flagella, and the arrangement of flagella were not important determinants of phylogeny. For example, Syntrophomonas wolfei has a gram-negative ultrastructure $(20)$ but turned out to be a member of the phylogenetically gram-positive phylum; furthermore, in the gram-positive phylum it was not closely related to the gram-negative subdivision containing the members of the Sporomusa-Selenomonas-Megasphaera group (33) (Fig. 4), which also have a gram-negative cell wall ultrastructure and some of which also have flagella laterally inserted into the concave side of the curved cells $(7,25,33)$, as Syntrophomonas wolfei does (20). As for the sporeformers FSM2, FSS7, and Syntrophospora bryantii, in the grampositive phylum they were not closely related to the Clos- tridium (low-G+C-content) subdivision, which is now the subdivision containing the genera Sporomusa and Megasphaera (Fig. 4), including sporeformers $(6,25,33)$.

The genus Syntrophomonas is the first genus of SFAS designated. Thus, we refer to the new group as the Syntrophomonas subdivision.

In an earlier study (28), the genus Syntrophomonas was placed in the family Bacteroidaceae in the gram-negative phylum. This placement is no longer valid because of the localization of this taxon in the gram-positive phylum. We recommend that the Syntrophomonas subdivision be placed in a new family, the Syntrophomonadaceae, which, after further phylogenetic studies are done, might become a new order in the gram-positive phylum of eubacteria. A description of the family follows.

Family Syntrophomonadaceae Zhao, Yang, Woese, and Bryant. Syntrophomonadaceae (Syn.tro.pho.mon.a.da'ce.ae. M.L. fem. n. Syntrophomonas, type genus of the family; -aceae, ending to denote a family; M.L. fem. pl. n. Syntrophomonadaceae, the Syntrophomonas family). Cells have various, often curved, rod shapes, with or without flagella and with or without endospores. Multiplication is by binary fission. Cells may have distinct outer membranes or no outer membranes and be either gram positive or gram negative. The members studied so far contain muramic and mesodiaminopimelic acids in their cell walls and contain poly- $\beta$ hydroxyalkanoates as reserve material.

These bacteria are heterotrophic; they are very strict anaerobes that $\beta$-oxidize saturated fatty acids containing 4 to 18 straight- or branched-chained carbon atoms, depending on the strain, with protons serving as the electron acceptor (i.e., $\mathrm{H}_{2}$ production). Because of the energetics of the reactions, the $\mathrm{H}_{2}$ must be maintained at a very low level, usually by hydrogenotrophic bacteria, for growth to occur (i.e., syntrophic metabolism). It is possible that formate in addition to $\mathbf{H}_{2}$ can serve as the interspecies electron carrier. Organic end products include acetate and other fatty acids, such as propionate, depending on the fatty acid energy source. With some energy sources that probably do not occur extracellularly in natural environments, members of the Syntrophomonadaceae can be grown in pure culture without an $\mathrm{H}_{2}$ scavenger because part of the substrate can be oxidized and part can be reduced without $\mathrm{H}_{2}$ production. These energy sources include compounds, such as crotonate, that can be dismutated to acetate and butyrate. Very long periods of "adaptation" may be required before metabolism of crotonate is initiated. Other compounds used as energy sources in pure cultures by at least some of these 
bacteria include trans-2-pentenoate, trans-2-hexenoate, trans-3-hexenoate, and trans,trans-2,4-hexadienoate. These compounds are reduced to saturated fatty acids such as $\mathrm{C}_{2}$ to $\mathrm{C}_{6}$ fatty acids such that little $\mathrm{H}_{2}$ (or formate) is produced (1). Carbohydrates, proteinaceous materials, alcohols, or other organic compounds do not support growth. Common electron acceptors such as fumarate, malate, nitrate, nitrite, $\mathrm{O}_{2}$, sulfate, sulfite, sulfur, and thiosulfate are not used.

Cells of the type species contain the usual enzymes for $\beta$-oxidation (22) but contain only acyl coenzyme A (coA): acetate-CoA transferases for $\mathrm{CoA}$ and no acyl-CoA synthetase. Butyrate-grown cells from cocultures do not contain CoA transferase activity for activation of crotonate, and the latter activity requires long adaptation. The cells contain phosphotransacetylase and acetate kinase for substrate level phosphorylation. The cells also contain a type $c$ cytochrome, and the cell yield for crotonate-grown cells is at least threefold greater than the yields for any other anaerobes that grow and dismutate crotonate to acetate and butyrate. This suggests that members of the genus Syntrophomonas may transfer energy via electron transport-linked reduction of crotonyl-CoA. None of the other crotonate-degrading species contains cytochromes (22).

These bacteria may be marine bacteria requiring at least $1 \% \mathrm{NaCl}$ and $0.15 \% \mathrm{MgCl}_{2} \cdot 6 \mathrm{H}_{2} \mathrm{O}$ or aquatic or anaerobic digestor sludge bacteria requiring considerably lower concentrations of these salts. Some require type $B$ vitamins, and some do not (6).

These bacteria are present in anaerobic microbial ecosystems, such as marine and freshwater sediments, sewage digestor sludge, and rumen digesta where organic matter is degraded with $\mathrm{CO}_{2}$ and $\mathrm{CH}_{4}$ as the major products.

Whether similar bacteria from thermophilic environments or other syntrophs such as those catabolizing benzoate or similar lowly substituted benzenes belong in this family is not yet known. A benzoate-catabolizing syntroph similar to Syntrophus buswellii was recently shown to adapt to growth on crotonate (19).

Phylogenetically, as determined by 16S rRNA sequencing, this family is in a so-far-unique group of gram-positive eubacteria. The strains studied so far have DNA $G+C$ contents ranging from 37 to $48 \mathrm{~mol} \%$.

The genus Syntrophomonas McInerney, Bryant, Hespell, and Costerton 1981 (20) emend. Lorowitz, Zhao, and Bryant 1989 (18) is the type genus.

Our comparative sequence analyses included most of the existing, documented SFAS. Four previously reported SFAS, three of which were mesophilic sporeformers $(31,39)$ and one of which was a thermophilic nonsporeformer (13), are no longer extant $(19,38,39)$ and thus are not available for sequencing. Syntrophomonas sapovorans (26) contains an unknown contaminating bacterium and therefore was not included in this comparative study. We were not familiar with the isovalerate-degrading syntroph of Stieb and Schink (35) until it was too late to include this organism in the study.

\section{ACKNOWLEDGMENTS}

We thank M. J. McInerney, W. E. C. Moore, J. M. Tiedje, and F. Widdel for supplying needed cultures. We are grateful to the Electron Microscopy Suite of the College of Veterinary Medicine, University of Illinois, for generously assisting our work with electron microscopy.

This research was supported by grant DE AC02 81 ER 10874 from the U.S. Department of Energy and by a grant from the Agricultural Experiment Station of the University of Illinois to M.P.B. and by grant NSF BSR 8705352 from the National Science Foundation to C.R.W.

\section{REFERENCES}

1. Amos, D. A., and M. J. McInerney. 1990. Growth of Syntrophomonas wolfei on unsaturated short chain fatty acids. Arch. Microbiol. 154:31-36.

2. Balch, W. E., and R. S. Wolfe. 1976. New approach to the cultivation of methanogenic bacteria: 2-mercaptoethanesulfonic acid (HS-CoM)-dependent growth of Methanobacterium ruminatium in a pressurized atmosphere. Appl. Environ. Microbiol. 32:781-791.

3. Balch, W. E., and R. S. Wolfe. 1979. Specificity and distribution of coenzyme M (2-mercaptoethanesulfonic acid). J. Bacteriol. 137:256-263.

4. Basgall, E. J., G. Scherba, and H. B. Gelberg. 1988. Diagnostic virology in veterinary pathology: techniques for negative staining, p. 366-367. In G. W. Bailey (ed.), Proceedings of the 46th Annual Meeting of the Electron Microscopy Society of America. San Francisco Press, Inc., San Francisco.

5. Beaty, P. S., and M. J. McInerney. 1987. Growth of Syntrophomonas wolfei in pure culture on crotonate. Arch. Microbiol. 147:389-393.

6. Beaty, P. S., and M. J. McInerney. 1990. Nutritional features of Syntrophomonas wolfei. Appl. Environ. Microbiol. 56:32233224.

7. Breznak, J. A., J. M. Switzer, and H. J. Seitz. 1988. Sporomusa termitida sp. nov., an $\mathrm{H}_{2} / \mathrm{CO}_{2}$-utilizing acetogen isolated from termites. Arch. Microbiol. 150:282-288.

8. Bryant, M. P. 1972. Commentary on the Hungate technique for culture of anaerobic bacteria. Am. J. Clin. Nutr. 25:1324-1328.

9. Bryant, M. P., and L. A. Burkey. 1953. Culture methods and some characteristics of some of the more numerous groups of bacteria in the bovine rumen. J. Dairy Sci. 36:205-217.

10. Canale, A., M. E. Valente, and A. Ciotti. 1984. Determination of volatile carboxylic acids $\left(\mathrm{C}_{1}-\mathrm{C}_{51}\right)$ and lactic acid in aqueous acid extracts of silage by high performance liquid chromatography. J. Sci. Food Agric. 35:1178-1182.

11. Cato, E. P., W. L. George, and S. M. Finegold. 1986. Endospore-forming Gram-positive rods and cocci. Genus Clostridium, p. 1141-1200. In P. H. A. Sneath, N. S. Mair, M. E. Sharpe, and J. G. Holt (ed.), Bergey's manual of systematic bacteriology, vol. 2. The Williams \& Wilkins Co., Baltimore.

12. De Soete, G. 1973. A least-squares algorithm for fitting additive trees to proximity data. Psychometrika 48:621-626.

13. Henson, J. M., and P. H. Smith. 1985. Isolation of a butyrateutilizing bacterium in coculture with Methanobacterium thermoautotrophicum from a thermophilic digester. Appl. Environ. Microbiol. 49:1461-1466.

14. Hungate, R. E. 1950 . The anaerobic mesophilic cellulolytic bacteria. Bacteriol. Rev. 14:1-41.

15. Jukes, T. H., and C. R. Cantor. 1969. Evolution of protein molecules, p. 21-132. In H. N. Munro (ed.), Mammalian protein metabolism. Academic Press, New York.

16. Lane, D. J., N. Pace, G. J. Olsen, D. A. Stahl, M. L. Sogin, and N. R. Pace. 1985. Rapid determination of 16 S ribosomal RNA sequences for phylogenetic analysis. Proc. Natl. Acad. Sci. USA 82:6955-6959.

17. Leedle, J. A. Z., and R. B. Hespell. 1980. Differential carbohydrate media and anaerobic replica plating techniques in delineating carbohydrate-utilizing subgroups in rumen bacteria populations. Appl. Environ. Microbiol. 29:709-719.

18. Lorowitz, W., H. Zhao, and M. P. Bryant. 1989. Syntrophomonas wolfei subsp. saponavida subsp. nov., a long-chain fatty acid-degrading anaerobic, syntrophic bacterium; Syntrophomonas wolfei subsp. wolfei subsp. nov.; and emended description of the genus and species. Int. J. Syst. Bacteriol. 39:122-126.

19. McInerney, M. J. Personal communication.

20. McInerney, M. J., M. P. Bryant, R. B. Hespell, and J. W. Costerton. 1981. Syntrophomonas wolfei gen. nov., sp. nov., an anaerobic syntrophic, fatty acid-oxidizing bacterium. Appl. Environ. Microbiol. 41:1029-1039.

21. McInerney, M. J., M. P. Bryant, and N. Pfennig. 1979. Anaerobic bacterium that degrades fatty acids in syntrophic association with methanogens. Arch. Microbiol. 122:129-135.

22. McInerney, M. J., and N. Q. Wofford. 1992. Enzymes involved 
in crotonate metabolism in Syntrophomonas wolfei. Arch. Microbiol. 158:344-349.

23. Miller, L. A. 1982. Practical rapid embedding procedure for transmission electron microscopy. Lab. Med. 13:752-756.

24. Miller, T. L., and M. J. Wolin. 1974. A serum bottle modification of the Hungate technique for cultivation of obligate anaerobes. Appl. Environ. Microbiol. 27:985-987.

25. Möller, B., R. Obmer, B. H. Howard, G. Gottschalk, and H. Hippe. 1984. Sporomusa, a new genus of Gram-negative anaerobic bacteria including Sporomusa sphaeroides sp. nov. and Sporomusa ovata sp. nov. Arch. Microbiol. 139:388-396.

26. Nisman, B. 1954. The Stickland reaction. Bacteriol. Rev. 18:1642.

27. Pfennig, N., and S. Wagener. 1986. An improved method of preparing wet mounts for photomicrographs of microorganisms. J. Microbiol. Methods 4:303-306.

28. Roy, R., E. Samin, H. C. Dubourgier, and G. Albangnai. 1986. Syntrophomonas sapovorans $\mathrm{sp}$. nov., a new obligately proton reducing anaerobe oxidizing saturated and unsaturated long chain fatty acids. Arch. Microbiol. 145:142-147.

29. Salanitro, J. P., and P. A. Muirhead. 1975. Quantitative method for gas chromatographic analysis of short-chain monocarboxylic and dicarboxylic acids in fermentation media. Appl. Environ. Microbiol. 29:374-381.

30. Sanger, T., S. Nicklen, and A. R. Coulson. 1977. DNA sequencing with chain-terminating inhibitors. Proc. Natl. Acad. Sci. USA 74:5463-5467.

31. Shelton, D. R., and J. M. Tiedje. 1984. Isolation and partial characterization of bacteria in an anerobic consortium that mineralizes 3-chlorobenzoic acid. Appl. Environ. Microbiol. 48:840-848.

32. Smith, R. L., and M. J. Klug. 1981. Electron donors utilized by sulfate-reducing bacteria in eutrophic lake sediments. Appl. Environ. Microbiol, 42:116-121.
33. Stackebrandt, E., H. Pohla, R. Kroppenstedt, H. Hippe, and C. W. Woese. 1985. 16S rRNA analysis of Sporomusa, Selenomonas, and Megasphaera: on the phylogenetic origin of grampositive eubacteria. Arch. Microbiol. 143:270-276.

34. Stieb, M., and B. Schink. 1985. Anaerobic oxidation of fatty acids by Clostridium bryantii sp. nov., a sporeforming, obligately syntrophic bacterium. Arch. Microbiol. 140:387-390.

35. Stieb, M., and B. Schink. 1986. Anaerobic degradation of isovalerate by a defined methanogenic coculture. Arch. Microbiol. 144:291-295.

36. Thauer, R. K., K. Jungermann, H. Henninger, J. Wenning, and K. Decker. 1968. The energy metabolism of Clostridium kluyveri. Eur. J. Biochem. 4:173-180.

37. Tiedje, J. M. Personal communication.

38. Tomei, F. A. Personal communication.

39. Tomei, F. A., J. S. Maki, and R. Mitchell. 1985. Interaction in syntrophic associations of endospore-forming, butyrate-degrading bacteria and $\mathrm{H}_{2}$-consuming bacteria. Appl. Environ. Microbiol. 50:1244-1250.

40. Widdel, F. 1988. Microbial ecology of sulfate- and sulfurreducing bacteria, p. 469-585. In A. J. B. Zender (ed.), Biology of anaerobic microorganisms. John Wiley and Sons, New York.

41. Woese, C. R. 1987. Bacterial evolution. Microbiol. Rev. 51:221271.

42. Woese, C. R., R. Gutell, R. Gupta, and H. F. Noller. 1983. Detailed analysis of the higher-order structure of $16 \mathrm{~S}$-like ribosomal ribonucleic acid. Microbiol. Rev. 47:621-669.

43. Yang, D., Y. Oyaizu, H. Oyaizu, G. J. Olsen, and C. R. Woese. 1985. Mitochondrial origins. Proc. Natl. Acad. Sci. USA 82: 4443-4447.

44. Zhao, H., D. Yang, C. R. Woese, and M. P. Bryant. 1989. Assignment of the syntrophic, fatty acid-degrading anerobe Clostridium bryantii to Syntrophospora bryantii gen. nov., comb. nov. Int. J. Syst. Bacteriol. 40:40-44. 